\title{
Suffizienz und Unternehmen - ein Paradox?
}

\author{
Alexandra Palzkill $\cdot$ Uwe Schneidewind
}

Online publiziert: 24. April 2015

(C) Springer-Verlag Berlin Heidelberg 2015

„Effizienz“ und „Wachstum“ scheinen die Imperative betriebswirtschaftlicher Theorie und Praxis zu sein. So lernen es Betriebswirtschaftsstudierende vom ersten Semester an und das erwarten Analysten, wenn börsennotierte Unternehmen ihre Quartalszahlen vorlegen. Unternehmen sollen wachsen - um Arbeitsplätze zu erhalten, um Gewinne zu erzielen, um im Wettbewerb zu bestehen. Aber sind diese Forderungen wirklich mit der Notwendigkeit eines ,immer Mehr“ verbunden? Die Ideen der Suffizienz, der Genügsamkeit, des Weniger scheinen auf den ersten Blick so wenig mit dem betrieblichen Alltag zu tun zu haben.

Doch was passiert, wenn die Welt der ökonomischen Effizienz und des ökonomischen Wachstums zunehmend an ökologische und auch soziale Grenzen stößt? Welche Rolle spielen Unternehmen in einem Umfeld, das mehr Suffizienz verlangt? Was bedeutet das für Geschäftsstrategien und die gesellschaftliche Rolle von Unternehmen?

Genau diesen Fragen möchte sich dieser Schwerpunkt nähern, indem er das Verhältnis von Suffizienz und Unternehmen ausleuchtet.

Spätestens seit dem Bericht des Club of Rome zu den „Grenzen des Wachstums“ (Meadows 1972) ist klar: in einer begrenzten Welt ist ein ewiges Mehr nicht möglich. Die Ressourcen und die ökologischen Senken, wie die Aufnahmekapazität von $\mathrm{CO}_{2}$ in der Atmosphäre, sind bereits heute überlastet - oder werden es bald sein. Um im ,safe operating space for humanity“ (Rockström 2009) zu bleiben, ist eine absolute Senkung des Ressourcenverbrauchs in vielen Dimensionen notwendig.

A. Palzkill $(\varangle) \cdot U$. Schneidewind

Wuppertal Institut für Klima, Umwelt, Energie,

Wuppertal, Deutschland

E-Mail: alexandra.palzkill@wupperinst.org
Unternehmen sind die Akteure, die Ressourcen gewinnund wohlstandsbringend in Güter und Dienstleistungen umwandeln - mit all den damit verbundenen ökologischen und sozialen Nebenfolgen. Um die Umweltbelastung zu senken, wird deswegen oftmals eine höhere Effizienz und Naturverträglichkeit von Seiten der Unternehmen gefordert - Suffizienz spielt in den Debatten höchstens auf Seiten der Konsumenten eine Rolle. Doch zeigt die Realität, dass die ökologischen Effekte einer effizienteren Produktion durch Rebound- und Wachstumseffekte beschränkt sind...Die absolute Umweltbelastung wird daher auch bei effizienterer Produktion bei steigendem Wirtschaftswachstum weiter steigen.

Aus diesem Grund haben Wachstumskritik und die Diskussion über Suffizienz in letzter Zeit stark an Bedeutung gewonnen. Die Bundestagsenquete „Wachstum, Wohlstand, Lebensqualität" (2011-2013), Initiativen alternativen Wirtschaftens wie der Transition-Town-Bewegung, oder die im September 2014 stattgefundene „De-Growth“-Konferenz in Leipzig mit über 3000 Teilnehmern sind Ausdruck davon. Doch auch in diesen Debatten findet sich meistens wenig zur betriebswirtschaftlichen Seite von Suffizienz oder Postwachstum. Dabei gibt es sie, die Ansätze von wachstumsneutralen oder suffizienten Unternehmen, wie die Beispiele in diesem Heft zeigen.

Wolfgans Sachs diskutiert einführend die Notwendigkeit von Suffizienz und zeigt auf, warum Regionalität und Entschleunigung sowie Gemeinwohlorientierung und Commons grundlegende Elemente einer suffizienteren Wirtschaftsweise sind. Er zeigt außerdem, dass Suffizienz weniger fremdbestimmter Verzicht als verantwortungsvolle Freiheit bedeutet, indem sie zur Kunst eines unabhängigeren Lebens wird.

Suffiziente Unternehmen stellen sich dabei grundsätzlich der Frage, wie die Befriedigung von Bedürfnissen über den 
Markt in Anbetracht dieser verantwortungsvollen Freiheit, geleistet werden kann.

Dass sich auch Produkt-Designer und Gestalter heute schon diesen Herausforderungen stellen, zeigen Christa Liedtke, Johannes Buhl und Alica Borgmann. Sie diskutieren die Notwendigkeit von suffizientem Design für suffizienteres Handeln, da die Gestaltung eines Produkts nicht nur die Produktion, sondern auch das gesamte Nutzerleben prägt - und festlegen, ob Suffizienz oder Verschwendung gefördert wird.

Melanie Lukas widmet sich einem suffizienteren Konsum. Er ist die Voraussetzung dafür, dass Geschäftsmodelle der Suffizienz entstehen können. Sie zeigt, wo Anknüpfungspunkte für suffizientes Handeln heute schon gegeben sind.

Michael Griese, Christel Kumbruck und Anne Schlichting beschreiben die Potenziale von Suffizienz auf persönlicher Ebene (individual und commercial) und zeigen die gesellschaftlichen Voraussetzungen (economic, legal) dafür auf. Am Beispiel der $\mathrm{CO}_{2}$-Reduktion in der Tourismusbranche werden die Möglichkeiten von Suffizienz diskutiert.

Dass gerade oftmals für kleine und mittlere Unternehmen „Nicht-Wachstum“ bereits Unternehmensrealität ist und damit Suffizienz Unternehmensstrategie werden kann, zeigen Jana Gebauer und Heike Mewes. Dabei wird deutlich, welche Gründe für ein Weniger bzw. Nicht-noch-mehr sprechen und wie diese Unternehmen trotzdem erfolgreich sind.

Henning Wilts erläutert in seinem Beitrag konkrete Möglichkeiten unternehmerischer Suffizienz am Beispiel der Abfallwirtschaft. Hier ist Suffizienz heute schon häufig ein funktionierender Business Case.
Anja Bierwirth widmet sich den Möglichkeiten von unternehmerischer Suffizienz im Gebäudebereich. Sie zeigt, wie suffiziente Wohnungsbauunternehmen lebensqualitätssteigernd und ökologisch agieren können.

Ein ökologisch ähnlich relevantes Feld thematisiert Dirk Posse in seinem Beitrag über Ernährung. Er diskutiert, welche Voraussetzungen für suffizienteres unternehmerisches Handeln im Nahrungsmittelbreich notwendig sind.

Wie eng suffiziente Geschäftsmodelle oftmals mit Social Entrepreneurship verknüpft sind, beschreiben Alexandra Palzkill, Matthias Wanner und Florian Markscheffel. Am Beispiel des Wuppertaler Social Business „Utopiastadt“ beleuchten sie ein oft wenig beachtetes Unternehmenssegment:

Grundsätzlich ist das Zusammenspiel von „Unternehmen und Suffizienz" heute noch ein Nischen-Phänomen. Die vorliegende uwf-Ausgabe macht deutlich, dass diese Nische jedoch schon größer ist, als auf den ersten Blick zu vermuten wäre. Der betriebswirtschaftlichen Forschung ist zu wünschen, dass sie künftig einen noch stärkeren Beitrag leistet, um die Rolle von Unternehmen bei der Transformation zu neuen Wohlstandsmodellen zu verstehen.

Alexandra Palzkill und Uwe Schneidewind

\section{Literatur}

Meadows DH et al (1972) The limits to growth: a report for the Club of Rome's project on the predicament of mankind. Universe Books, New York

Rockström J et al (2009) A safe operating space for humanity. Nature 461(7263):472-475 